\title{
Delays in switching patients onto second-line antiretroviral treatment at a public hospital in eThekwini, KwaZulu-Natal
}

\begin{tabular}{|c|c|}
\hline \multicolumn{2}{|c|}{$\begin{array}{l}\text { Authors: } \\
\text { Denver Narainsamy }{ }^{1} \\
\text { Saajida Mahomed }{ }^{2} \text { (D) }\end{array}$} \\
\hline \multicolumn{2}{|c|}{$\begin{array}{l}\text { Affiliations: } \\
{ }^{1} \text { School of Nursing and Public } \\
\text { Health, University of } \\
\text { KwaZulu-Natal, South Africa }\end{array}$} \\
\hline \multicolumn{2}{|c|}{$\begin{array}{l}{ }^{2} S c h o o l \text { of Laboratory } \\
\text { Medicine and Medical } \\
\text { Sciences, University of } \\
\text { KwaZulu-Natal, South Africa }\end{array}$} \\
\hline \multicolumn{2}{|c|}{$\begin{array}{l}\text { Corresponding author: } \\
\text { Denver Narainsamy, } \\
\text { denvernarainsamy@gmail. } \\
\text { com }\end{array}$} \\
\hline \multicolumn{2}{|c|}{$\begin{array}{l}\text { Dates: } \\
\text { Received: } 23 \text { Sept. } 2016 \\
\text { Accepted: } 05 \text { Feb. } 2017 \\
\text { Published: } 31 \text { Mar. } 2017\end{array}$} \\
\hline \multicolumn{2}{|c|}{$\begin{array}{l}\text { How to cite this article: } \\
\text { Narainsamy D. Mahomed S. } \\
\text { Delays in switching patients } \\
\text { onto second-line } \\
\text { antiretroviral treatment at a } \\
\text { public hospital in eThekwini, } \\
\text { KwaZulu-Natal. S Afr J HIV } \\
\text { Med. 2017;18(1), a696. } \\
\text { https://doi.org/10.4102/ } \\
\text { sajhivmed.v18i1.696 }\end{array}$} \\
\hline \multicolumn{2}{|c|}{$\begin{array}{l}\text { Copyright: } \\
\text { (c) 2017. The Authors. } \\
\text { Licensee: AOSIS. This } \\
\text { is licensed under the } \\
\text { Creative Commons } \\
\text { Attribution License. }\end{array}$} \\
\hline 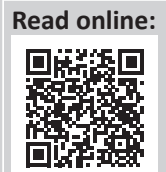 & $\begin{array}{l}\text { Scan this QR } \\
\text { code with your } \\
\text { smart phone or } \\
\text { mobile device } \\
\text { to read online. }\end{array}$ \\
\hline
\end{tabular}

Background: South Africa has one of the largest antiretroviral treatment (ART) programmes globally. In addition to increasing access to ART, it is important that the health system also focuses on the appropriate management of patients who fail first-line ART. Delays in switching patients onto second-line ART can adversely affect patient outcomes.

Aim: To identify the patient-related and programmatic factors that delay switching patients onto second-line ART, and to assess whether these delays contribute to subsequent virological failure.

Methods: Clinical records of adult patients switched onto second-line ART between 2011 and 2014 at a public antiretroviral clinic were used to collect demographic, clinical, laboratory and programmatic data (availability of viral load results, inadequate patient follow-up, insufficient notes for effective follow-up). Data were analysed using univariate and multivariate logistic regression.

Results: The median duration from the date of first and confirmatory documented high viral load (VL > 1000 copies $/ \mathrm{mL}$ ) to being switched to second-line ART was 13.2 months [interquartile range (IQR) 1.1-52.7 months] and 6.4 months (IQR 0-43.3 months), respectively. Inadequate prescriber notes for appropriate follow-up $(p=0.01)$ and unavailability of patients' viral load results $(p=0.02)$ were significantly associated with delays in switching to secondline ART. There was no significant association between the time taken to switch to second-line ART and subsequent virological failure.

Conclusion: We observed lengthy delays in switching patients to second-line ART. Modifiable programmatic factors were found to be significantly associated with delays in switching to second-line ART.

\section{Introduction}

In 2011, it was estimated that more than eight million people were accessing antiretroviral treatment (ART) in low- and middle-income countries. ${ }^{1}$ This rapid scale-up of ART initiation at a global level has significantly improved health outcomes for the majority of those living with human immunodeficiency virus (HIV) and acquired immunodeficiency syndrome (AIDS). However, the critical public health challenge must now shift to managing the increasing number of patients that are likely to fail first-line ART. ${ }^{2}$

South Africa has one of the largest ART programmes globally with more than three million people currently accessing treatment. In addition to having the largest HIV epidemic worldwide, with an estimated 6.2 million people infected, ${ }^{3}$ South Africa has a large burden of communicable diseases, non-communicable diseases, violence and injury, and perinatal and maternal diseases. ${ }^{4}$ These challenges have conveyed significant pressures on the delivery of healthcare within the country.

The current National ART Guidelines advocate an early switch to second-line ART in the face of virological failure. Two consecutive viral loads (VLs), taken two months apart, that are greater than 1000 copies / $\mathrm{mL}$ serve as confirmation of virological failure, provided that factors related to adherence, drug toxicities and drug interactions are adequately addressed. ${ }^{5}$ Ideally, an adult patient should be switched to second-line ART within a period of three months from the date of first documented high VL. However, limited research has demonstrated delays in switching patients to second-line ART. 2,6 
Although theoretical principles suggest that the delay in switching patients to second-line ART can result in the accumulation of resistant mutations that may compromise second-line $\mathrm{ART}^{7}$ this is unlikely in the context of the South African ART programme as cross-resistance between first-line and second-line drugs are unlikely. ${ }^{8}$ However, limited research from South Africa and other African countries have shown that patients maintained on a failing first-line regimen for long periods had an increased risk of mortality after switching to a protease inhibitor (PI)-based second-line regimen. ${ }^{9,10}$ These findings highlight the importance of timely switching of patients who are failing on first-line ART to second-line ART. We investigated the programmatic and patient-related factors that contribute to delays in switching patients to second-line ART and assessed the association between these delays and subsequent virological suppression.

\section{Methods}

\section{Study setting and population}

The study was conducted at an outpatient antiretroviral clinic situated in a public hospital in the eThekwini Health District, KwaZulu-Natal (KZN), South Africa. The area consists of a peri-urban setting with more than 6500 patients accessing ART services at this hospital in 2014.

Adult patients ( $\geq 18$ years) attending the clinic who were on a non-nucleoside reverse transcriptase inhibitor (NNRTI)based first-line ART regimen and switched onto a PI-based second-line ART regimen between January 2011 and January 2014 as a result of virological failure were included in the study. These patients were identified using pharmacy records, ${ }^{1}$ electronic databases ${ }^{2}$ and active patient identification during prescription refills. Patients who had been referred from primary healthcare clinics owing to virological failure were excluded from the study as their medical records were not complete.

\section{Study design and data collection}

A retrospective cohort study design was used. Patient's medical records were accessed to obtain demographic, clinical and programmatic data. The data were collected with the assistance of a trained research nurse using a standardised data collection tool. Clinical variables recorded included baseline CD4+ count, baseline VL, first-line ART regimen, regimen modifications prior to switch to second-line ART (changes made to the standard first-line ART regimen owing to adverse drug reactions or drug interactions), date of first and second documented high VL, date of switch to secondline ART, VL at switch to second-line ART and VL at least 12 months after switch to second-line ART. Pregnancy and tuberculosis co-infection were also recorded. Patient-related variables included age, gender and patients defaulting their appointment (defined as patient failing to attend the clinic within 30 days of their set appointment date during the period of first documented high VL and switch to second-line ART). The programmatic variables recorded included the availability of VL results, inadequate prescriber notes for appropriate follow-up (defined as the attending clinician failing to document a management plan in the patient's medical record from the date of first documented high VL, by either not referring the patient for step-up adherence counselling, not documenting the need for a repeat VL after two months and not documenting the high VL in the patient's medical record), inadequate prescriber follow-up at the subsequent visit (defined as the attending clinician failing to follow the patient's clinical management plan of the previous visit by either not repeating a VL test or not switching secondline ART after reviewing the repeated VL result) and the patient's medical record being mislaid. Time from first and second documented high VLs to time of switch to secondline ART was measured and the VL measurement at least one year after switch to second-line ART was recorded.

\section{Statistical analysis}

The association between delays in switching to second-line ART and subsequent virological failure was determined using incidence risk ratios with 95\% confidence limits and inferential statistical analysis using Pearson's chi-square tests. Multivariate analysis (Poisson regression) was performed to determine if any of the patients or programmatic variables was independently associated with the development of virological failure. All statistical analyses were performed using STATA version 13 (Stata Corporation, College Station, Texas, USA).

\section{Ethical considerations}

The research was approved by the Biomedical Research and Ethics Committee of the University of KwaZulu-Natal (BE $337 / 14)$. Gatekeeper permission was obtained from the hospital's Chief Executive Officer and the KwaZulu-Natal Provincial Health Research and Ethics committee.

\section{Results}

A total of 238 patients were switched to second-line ART during the three-year period. Fifteen patients were excluded because of missing data (Figure 1). Of the remaining 223 patients, $68 \%(n=152)$ were female (Table 1$)$. The median age for males and females was 42 [interquartile range (IQR)

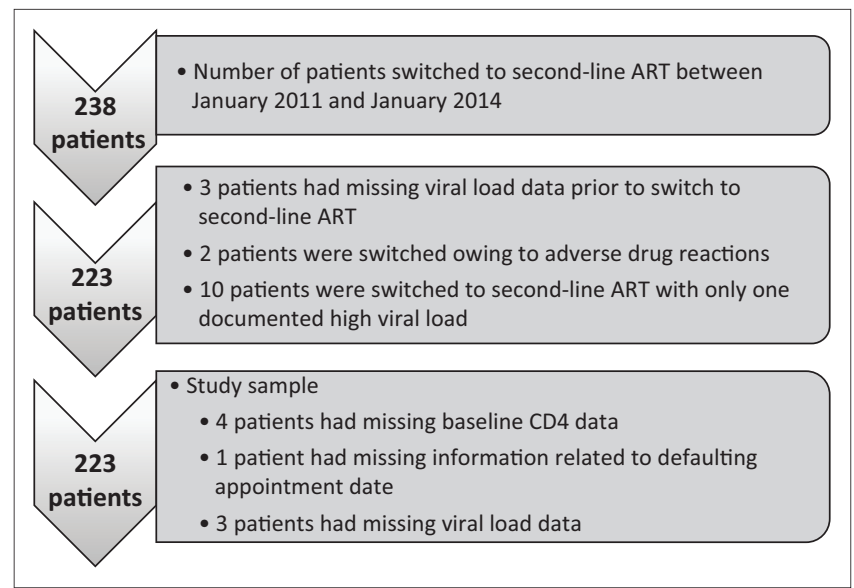

FIGURE 1: Summary of study sample. 
TABLE 1: Characteristics of patients switched to second-line antiretroviral treatment at an antiretroviral clinic in eThekwini Health District, 2011 to 2014.

\begin{tabular}{|c|c|c|c|}
\hline Patient characteristic & Variable & $n$ & $(\%)$ \\
\hline \multirow[t]{2}{*}{ Gender } & Male & 71 & 31.9 \\
\hline & Female & 152 & 68.1 \\
\hline \multirow[t]{4}{*}{ Age (years) } & $18-29$ & 35 & 15.7 \\
\hline & $30-39$ & 103 & 46.2 \\
\hline & $40-49$ & 66 & 29.6 \\
\hline & $>49$ & 19 & 8.5 \\
\hline \multirow[t]{3}{*}{ Baseline $\mathrm{CD} 4$ count (cells $/ \mathrm{mm}^{3}$ ) } & $0-100$ & 131 & 59.8 \\
\hline & $101-200$ & 65 & 29.7 \\
\hline & $>200$ & 23 & 10.5 \\
\hline History of TB & Yes & 73 & 33.2 \\
\hline $\begin{array}{l}\text { Pregnant (during period of } \\
\text { virological failure) }\end{array}$ & Yes & 21 & 14 \\
\hline \multirow[t]{7}{*}{ First-line ART exposure ever } & 3TC-TDF-EFV & 68 & 30.5 \\
\hline & 3TC-TDF-NVP & 43 & 19.3 \\
\hline & 3TC-AZT-EFV & 7 & 3.1 \\
\hline & 3TC-AZT-NVP & 8 & 3.6 \\
\hline & 3TC-D4T-EFV & 67 & 26 \\
\hline & 3TC-D4T-NVP & 58 & 26 \\
\hline & 3TC-ABC-EFV & 1 & 0.4 \\
\hline \multirow[t]{7}{*}{ First-line ART exposure at switch } & 3TC-TDF-EFV & 68 & 30.5 \\
\hline & 3TC-TDF-NVP & 40 & 17.9 \\
\hline & 3TC-AZT-EFV & 6 & 2.7 \\
\hline & 3TC-AZT-NVP & 8 & 3.5 \\
\hline & 3TC-D4T-EFV & 55 & 24.6 \\
\hline & 3TC-D4T-NVP & 45 & 20.2 \\
\hline & 3TC-ABC-EFV & 1 & 0.4 \\
\hline $\begin{array}{l}\text { Regimen modification prior } \\
\text { to switch }\end{array}$ & Yes & 29 & 13 \\
\hline $\begin{array}{l}\text { Patient defaulting their } \\
\text { appointment }\end{array}$ & Yes & 38 & 17.1 \\
\hline Blood results available (VL) & Yes & 196 & 87.9 \\
\hline $\begin{array}{l}\text { Inadequate prescriber notes for } \\
\text { effective follow-up }\end{array}$ & Yes & 126 & 56.5 \\
\hline $\begin{array}{l}\text { Inadequate prescriber follow-up } \\
\text { at subsequent visit }\end{array}$ & Yes & 166 & 74.4 \\
\hline $\begin{array}{l}\text { Patients medical records lost } \\
\text { or misplaced }\end{array}$ & Yes & 9 & 4 \\
\hline \multirow[t]{3}{*}{ Viral load at switch (copies/mL) } & $<10000$ & 45 & 20.2 \\
\hline & $10000-100000$ & 117 & 52.5 \\
\hline & $>100000$ & 61 & 27.3 \\
\hline
\end{tabular}

$\mathrm{TB}$, tuberculosis; ART, antiretroviral treatment; VL, viral load; TDF, tenofovir; EFV, efavirenz.

19-73 years] and 36 years (IQR 18-57), respectively $(p<0.01)$. The median baseline CD4 count of patients was 94 cells $/ \mathrm{mm}^{3}$ (IQR 2-384).

\section{Delays in switching to second-line antiretroviral treatment}

The median duration from the date of first documented high VL and switch to second-line ART was 13.2 months (IQR 1.1-52.7 months), and the median duration from the time of confirmed virological failure (second documented high VL) and switch to second-line ART was 6.4 months (IQR 0-43.3 months). The median time interval between first and second documented high VL was 6.8 months (IQR 0.1-32 months). Eighty patients (36\%) experienced delays of greater than six months from the date of confirmed virological failure to the date of switch to second-line ART.

In bivariate analysis, there were no significant associations between the clinical or patient-related factors and delays
TABLE 2: Patient-related and programmatic variables associated with delays in switching patients to second-line antiretroviral treatment at an antiretroviral clinic in eThekwini Health District, 2011 to 2014.

\begin{tabular}{|c|c|c|c|c|}
\hline Factor & $\mathrm{RR}(95 \% \mathrm{Cl})$ & $p$-value & Adjusted RR $(95 \% \mathrm{Cl})$ & $p$-value \\
\hline Gender (females) & $1.2(0.8-1.9)$ & 0.29 & $0.2(0.002-27)$ & 0.33 \\
\hline \multicolumn{5}{|l|}{ Age range (years) } \\
\hline $18-29$ & $1.1(0.5-2.4)$ & 0.84 & $0.9(0.4-2.0)$ & 0.80 \\
\hline $30-39$ & $1.3(0.6-2.6)$ & 0.48 & $1.1(0.6-2.3)$ & 0.73 \\
\hline $40-49$ & $0.9(0.4-2.0)$ & 0.92 & $0.8(0.4-1.6)$ & 0.47 \\
\hline $\begin{array}{l}\text { Patient defaulted } \\
\text { appointment }\end{array}$ & $1.3(0.9-2.0)$ & 0.19 & $1.4(0.9-2.5)$ & 0.21 \\
\hline $\begin{array}{l}\text { Blood results } \\
\text { unavailable }\end{array}$ & $1.4(0.9-2.2)$ & 0.13 & $1.7(1.1-2.6)$ & 0.02 \\
\hline $\begin{array}{l}\text { Insufficient notes for } \\
\text { follow-up }\end{array}$ & $1.8(1.2-2.7)$ & 0.004 & $1.9(1.2-3.2)$ & 0.01 \\
\hline $\begin{array}{l}\text { Inadequate patient } \\
\text { follow-up }\end{array}$ & $1.4(0.9-2.2)$ & 0.18 & $1.1(0.6-1.9)$ & 0.79 \\
\hline Patients records lost & $1.3(0.6-2.7)$ & 0.56 & $1.2(0.5-2.7)$ & 0.70 \\
\hline
\end{tabular}

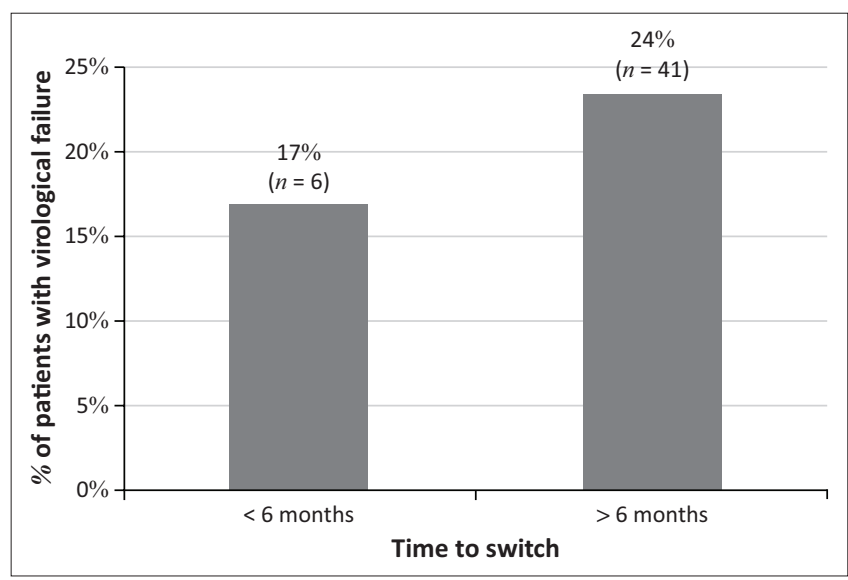

FIGURE 2: Virological failure among patients switched to second-line antiretroviral treatment at an antiretroviral clinic in eThekwini health district, 2011 to 2014.

in switching to second-line ART. Patients whose medical records contained insufficient prescriber notes for appropriate follow-up demonstrated a 1.8-fold higher risk of experiencing delays in switching to second-line ART (95\% CI, 1.21-2.68, $p=0.004$ ) when compared to patients whose medical records had sufficient prescriber notes. In multivariate analysis, both insufficient prescriber notes for appropriate follow-up (RR 1.94, 95\% CI, 1.16-3.24, $p=0.01$ ) and non-availability of VL results (RR 1.66, 95\% CI, $1.08-2.56, p=0.02$ ) were significantly associated with delays in switching to second-line ART (Table 2).

\section{Virological failure}

Of the 223 patients in the cohort, almost a quarter (21\%, $n=47$ ) developed virological failure within 18 months of being switched to second-line ART. The majority of these patients $(87 \%, n=41)$ had more than a six-month delay to switching to second-line ART from the date of first documented high VL. (Figure 2). Patients with delays greater than six months demonstrated a 1.6-fold higher risk of developing virological failure when compared to patients switched within six months (95\% CI, 0.71-3.54, $p=0.26$ ). There was no significant linear correlation between the time taken to switch to second-line ART and virological 
failure $(r=0.027)$. In bivariate analysis, patients that defaulted their appointment dates demonstrated a 1.9-fold higher risk of virological failure compared to patients that did not default their appointment dates (95\% CI, 1.10-3.21, $p=0.02$ ). However, in multivariate analysis, this association was not significant $(p=0.11)$ (Table 3$)$.

Patients in the age group 18-29 years had a 1.9-fold higher risk of developing virological failure when compared to patients in the oldest age category (95\% CI, 0.63-6.20, $p=0.24)$.

\section{Discussion}

Our study is one of the few local studies that investigated programmatic and patient-related factors that could delay switching patients to second-line ART. The median time taken to switch patients onto second-line ART (6.4 months from the date of confirmed documented high VL) is greater than the reported median time in other studies conducted within South Africa. In Khayelistha, Cape Town, the average time taken to switch patients to second-line ART was 5.3 months (IQR 2.2-11.2). ${ }^{6}$ A large, multi-centre study conducted across five sites in Durban, Cape Town and Gauteng reported an average time of 4.6 months (IQR 2.1-8.7) before patients were switched to second-line ART. ${ }^{2}$ The median time between the first and second documented high viral was 6.8 months which is longer than the two months time advocated in the National ART Guidelines. ${ }^{5}$

We identified significant associations between modifiable programmatic factors and the delay in switching patients to second-line ART. The unavailability of VL results delays clinical decision-making and can potentially compromise patient care. This finding highlights the need for information technology and telecommunication networks to support healthcare delivery within ART clinics in KZN.

Insufficient prescriber notes is a concern, especially as patients often do not see the same healthcare provider on

TABLE 3: Programmatic, patient-related and clinical variables associated with virological failure at an antiretroviral clinic in eThekwini Health District, 2011 to 2014

\begin{tabular}{|c|c|c|c|c|}
\hline Factor & $\mathrm{RR}(95 \% \mathrm{Cl})$ & $p$ & Adjusted RR (95\% Cl) & $p$ \\
\hline Gender (females) & $0.8(0.5-1.4)$ & 0.47 & $0.08(<0.01-404)$ & 0.56 \\
\hline Age range (years) & - & - & - & - \\
\hline $18-29$ & $1.5(0.6-4.1)$ & 0.37 & $1.9(0.6-6.2)$ & 0.24 \\
\hline $30-39$ & $0.8(0.3-2.1)$ & 0.64 & $0.9(0.3-2.7)$ & 0.87 \\
\hline $40-49$ & $0.9(0.3-2.4)$ & 0.86 & $0.9(0.3-2.7)$ & 0.85 \\
\hline Viral load at switch & $1.1(0.9-1.3)$ & 0.45 & $1.1(0.8-1.7)$ & 0.53 \\
\hline History of TB & $0.9(0.6-1.6)$ & 0.84 & $0.8(0.5-1.4)$ & 0.40 \\
\hline Pregnancy & $1.0(0.9-1.1)$ & 0.49 & $0.9(0.3-2.9)$ & 0.92 \\
\hline $\begin{array}{l}\text { Regimen modification } \\
\text { prior to switch }\end{array}$ & $0.9(0.5-2.1)$ & 0.93 & $0.9(0.4-2.0)$ & 0.83 \\
\hline First-line regimen & - & - & - & - \\
\hline $\begin{array}{l}\text { Exposure to EFV-based } \\
\text { first-line regimen }\end{array}$ & $1.3(0.8-2.3)$ & 0.29 & $1.4(0.8-2.5)$ & 0.28 \\
\hline $\begin{array}{l}\text { Delays of greater than } \\
\text { six months }\end{array}$ & $1.7(0.8-3.8)$ & 0.18 & $1.6(0.7-3.5)$ & 0.26 \\
\hline $\begin{array}{l}\text { Patients defaulted } \\
\text { appointment }\end{array}$ & $1.9(1.1-3.2)$ & 0.02 & $1.5(0.9-2.7)$ & 0.11 \\
\hline
\end{tabular}

TB, tuberculosis; EFV, efavirenz. their follow-up visit. The poor documentation of a comprehensive patient treatment plan or inadequate patient follow-up in the face of an available treatment plan is an avoidable occurrence that can compromise patient care. These factors, together with the delays in confirming virological failure and switching patients to second-line ART, highlight the absence of robust systems necessary to manage chronic diseases like HIV within the public healthcare setting in South Africa. Studies within Africa have demonstrated that only $2.4 \%$ of patients are switched to second-line ART, ${ }^{11}$ in contrast to the World Health Organization's estimates that $9.4 \%$ of patients are likely to fail first-line ART after 12 months on treatment. ${ }^{12}$

The South African ART programme does not allow for genotypic testing at first-line ART failure; this therefore limits our interpretation of the virological failure seen in $21 \%$ of the cohort after switching to second-line ART. We observed no significant association between delays in switching patients to second-line ART and subsequent virological failure. Although studies demonstrate that maintaining a patient on a failing first-line regimen for prolonged periods may lead to the accumulation of complex mutations that would compromise the nucleoside reverse transcriptase inhibitor (NRTI) backbone of second-line $\mathrm{ART}^{8,13}$ this appears to have less impact in South Africa. This finding is consistent with the current literature which demonstrates that the majority of resistance patterns seen at first-line ART failure does not confer resistance to South Africa's second-line ART drugs. ${ }^{8}$ In addition, studies have documented very low rates of drug resistance to a proteasebased second-line regimen. ${ }^{14}$

We observed that patients who defaulted their appointment dates (a surrogate marker for adherence) demonstrated a higher risk of virological failure when compared to patients that did not. Although this increased risk was not significant, when combined with the short follow-up period within the study (less than 18 months after switch to second-line ART), the most plausible reason for second-line virological failure points to issues of adherence rather than drug resistance.

Although the increased risk of virological failure amongst younger patients was not statistically significant, this finding supports the available body of evidence which suggests that patients in younger age groups demonstrate poor adherence to ART when compared to older patients, subsequently making them more susceptible to drug resistance and virological failure. ${ }^{15}$

The study limitations were related to the retrospective nature of the study design. Patients with virological failure but not switched onto second-line ART owing to being transferred to another healthcare facility, defaulting or death were excluded. The use of data of patients defaulting their appointment dates is not an accurate reflection of adherence to treatment. We assumed that virological failure to second-line ART was acquired as it was not possible to determine if a patient was 
infected with a resistant strain of HIV. However, it is unlikely that transmitted resistance would have affected the study results given the low rates of transmitted resistance to NNRTIs, with little or no transmitted resistance documented for PIs. ${ }^{16,17}$ To assess the delays in switching patients to second-line ART, we looked at the clinician's documentation of a high VL and the availability of VL results in patients' medical records. It is possible that a patient's VL result may have been available but not documented or filed in the patient's medical record. This may have either overestimated or underestimated the time taken to switch to second-line treatment. We were unable to analyse patients' baseline VL results in relation to virological failure as changes to the South African ART guidelines in 2012 eliminated the need for baseline VLs when initiating patients onto ART. ${ }^{5}$ Although the study was limited to one antiretroviral clinic in eThekwini Health District, it is likely to represent many other antiretroviral clinics in the district as most of the clinics face similar challenges. In more rural settings, the delay in switching patients to second-line ART is likely to be longer as a result of the reduced availability of skilled human resources ${ }^{18,19}$ and the absence of telecommunication networks to support clinical decision-making.

\section{Conclusion and recommendations}

Our study demonstrated considerable delays in switching patients to second-line ART. The programmatic factors associated with these delays are preventable. Strengthening documentation and record keeping through the training of healthcare professionals remains essential in addressing the gaps associated with chronic disease management, in addition to alleviating the medico-legal implications of such practices. Upgrading and integrating of the information technology infrastructure within the public healthcare sector will allow for a more confident and responsive healthcare system that will improve the clinical management of patients.

Further investigation into the long-term outcomes of patients with delayed switching to second-line ART is warranted, with emphasis on more accurate methods of assessing patient adherence.

\section{Acknowledgements}

The authors would like to acknowledge Prof. Benn Sartorius for his assistance with the statistical analysis and Dr Stephen Knight for reviewing the article in preparation for publication, both from the Discipline of Public Health Medicine at the University of KwaZulu-Natal.

\section{Competing interests}

All authors declare that they do not have any conflict of interest. A journal author declaration and publishing license have been submitted by the main author on behalf of all contributing authors.

\section{Authors' contributions}

D.N. was the lead researcher, conceptualised the research project, performed data collection with the assistance of a trained research nurse, analysed the data with the assistance of a biostatistician and wrote all versions of the article. S.M. assisted in refining the research concept and study methods, supervised the data analysis and reviewed all versions of the article.

\section{References}

1. World Health Organization. Progress report 2011. Global HIV/AIDS response: Epidemic update and health sector progress towards universal access [homepage on the Internet]. Geneva: World Health Organization; 2011 [cited 2013 Mar 24]. Available from: http://www.who.int.hiv.pub.progress_report2011/en/index.html

2. Fox MP, Cutsem GV, Giddy J, et al. Rates and predictors of failure of first-line antiretroviral therapy and switch to second-line art in South Africa. J Acquir Immune Defic Syndr. 2012;60:428-437. https://doi.org/10.1097/qai.0b013e318 2557785

3. Statistics South Africa. Mid-year population estimates, 2015 [homepage on the Internet]. Pretoria: The Republic of South Africa; 2015 [cited 2016 Oct 9]. Available from: http://www.statssa.gov.za/publications/P0302/P03022015.pdf

4. Mayosi BM, Lawn JE, Van Niekerk A, et al. Health in South Africa: Changes and challenges since 2009. Lancet. 2012;380:2029-2043. https://doi.org/10.1016/ S0140-6736(12)61814-5

5. Department of Health. The Republic of South Africa. The South African antiretroviral treatment guidelines, 2013 [homepage on the Internet]. Pretoria: The Department of Health; 2013 [cited 2014 Apr 13]. Available from: http://www. Department of Health; 2013 [cited 2014 Apr 13]. Avil
kznhealth.gov.za/medicine/2013_ART_Guidelines.pdf

6. Boulle A, Van Cutsem G, Hilderbrand K, et al. Seven-year experience of a primary care antiretroviral treatment programme in Khayelitsha, South Africa. AIDS 2010;24:563-572. https://doi.org/10.1097/QAD.0b013e328333bfb7

7. Gilks CF, Crowley S, Ekpini R, et al. The WHO public-health approach to antiretroviral treatment against HIV in resource-limited settings. Lancet. 2006;368:505-510. https://doi.org/10.1016/S0140-6736(06)69158-7

8. Hosseinipour M, Gupta RK, Van ZyI G, Eron JJ, Nachega JB. Emergence of HIV drug resistance during first- and second-line antiretroviral therapy in resource-limited settings. J Infect Dis. 2013;207(Suppl 2):S49-S56.

9. Petersen ML, Tran L, Geng EH, et al. Delayed switch of antiretroviral therapy after virologic failure associated with elevated mortality among HIV-infected adults in Africa. AIDS. 2014;28(14):2097-2107. https://doi.org/10.1097/QAD.000000 0000000349

10. Gsponer T, Petersen M, Egger M, et al. The causal effect of switching to second-line ART in programmes without access to routine viral load monitoring. AIDS 2012;26(1):57-65. https://doi.org/10.1097/QAD.0b013e32834e1b5f

11. Madec $Y$, Leroy S, Rey-Cuille MA, Huber F, Calmy A. Persistent difficulties in switching to second-line ART in sub Saharan Africa - A systematic review and meta-analysis. PLoS One. 2013;8(12):e82724. https://doi.org/10.1371/journal. pone.0082724

12. World Health Organization. The HIV drug resistance report - 2012 [homepage on the Internet]. Geneva, Switzerland: World Health Organization; 2012 [cited 2014 Mar 2]. Available from: http://www.who.int/entity/hiv/pub/drugresistance/ report2012/en/index.html

13. Gupta RK, Pillay D. HIV resistance and the developing world. Int J Antimicrob Agents. 2007;29:510-517. https://doi.org/10.1016/j.ijantimicag.2007.01.003

14. Olawale A, Mookerjeeb S, Edward J. Treatment outcomes of patients on secondline antiretroviral therapy in resource-limited settings: A systematic review and meta-analysis. AIDS. 2012;26:929-938. https://doi.org/10.1097/QAD.0b013e32 $8351 \mathrm{f5b} 2$

15. Hinkin $\mathrm{CH}$, Hardy DJ, Mason $\mathrm{KI}$, et al. Medication adherence in HIV-infected adults: Effect of patient age, cognitive status, and substance abuse. AIDS. 2004;18(1): S19-S25. https://doi.org/10.1097/00002030-200418001-00004

16. World Health Organization. The World Health Organization's global strategy for prevention and assessment of HIV resistance homepage on the Internet]. Geneva, Switzerland: World Health Organization; 2008 [cited 2014 Mar 2]. Available from: http://www.who.int/hiv/drugresistance/WHO_HIVDR strategy. pdf?ua $=1$

17. Baxter JD, Dunn D, White E, et al. Global HIV-1 transmitted drug resistance in the INSIGHT Strategic Timing of Antiretroviral Treatment (START) trial. HIV Med. 2015;16(Suppl 1):77-87. https://doi.org/10.1111/hiv.12236

18. Strasser R. Rural health around the world: Challenges and solutions. Fam Pract [serial on the Internet]. 2003 [cited 2016 Sep 16];20(4):457-463. Available from: http://fampra.oxfordjournals.org/content/20/4/457.full.pdf+html

19. Lehmann U, Dieleman M, Martineau T. Staffing remote rural areas in middle- and low-income countries: A literature review of attraction and retention. BMC Health Serv Res [serial on the Internet]. 2008 [cited 2016 Sep 16];8:19. Available from: $\mathrm{http}: / /$ bmchealthservres.biomedcentral.com/articles/10.1186/1472-6963-8-19 